\title{
Спонтанное и стимулированное излучение двухчастотного квантово-каскадного лазера
}

\author{
(C) А.В. Бабичев ${ }^{1,2}$, А.Г. Гладышев ${ }^{1,2}$, А.С. Курочкин ${ }^{1}$, Е.С. Колодезный ${ }^{1}$, \\ В.Н. Неведомский ${ }^{3}$, Л.Я. Карачинский ${ }^{1,2,3}$, И.И. Новиков ${ }^{1,2,3}$, \\ А.Н. Софронов ${ }^{4}$, А.Ю. Егоров ${ }^{1,2}$ \\ ${ }^{1}$ Санкт-Петербургский национальный исследовательский университет \\ информационных технологий, механики и оптики, \\ 197101 Санкт-Петербург, Россия \\ ${ }^{2}$ ООО „Коннектор Оптикс“, \\ 194292 Санкт-Петербург, Россия \\ ${ }^{3}$ Физико-технический институт им. А.Ф. Иоффе Российской академии наук, \\ 194021 Санкт-Петербург, Россия \\ ${ }^{4}$ Санкт-Петербургский политехнический университет Петра Великого, \\ 195251 Санкт-Петербург, Россия \\ E-mail: andrey.babichev@connector-optics.com; Researcher ID: F-6479-2015
}

(Получена 29 октября 2018 г. Принята к печати 5 ноября 2018 г.)

\begin{abstract}
Представлены результаты экспериментов по изготовлению методом молекулярно-пучковой эпитаксии и исследованию гетероструктур двухчастотного квантово-каскадного лазера, изготовленных на основе гетеропары твердых растворов $\operatorname{In}_{0.53} \mathrm{Ga}_{0.47} \mathrm{As} / \mathrm{Al}_{0.48} \mathrm{In}_{0.52} \mathrm{As}$ на подложке InP. Гетероструктуры содержали квантовые каскады, излучающие на длине волны 9.6 мкм, и каскады, которые излучают на длине волны 7.6 мкм. Показано высокое структурное совершенство изготовленных гетероструктур. Проведено исследование спектров спонтанного и стимулированного излучения, продемонстрирована многомодовая лазерная генерация полосковых лазеров на длине волны излучения 7.6 мкм.
\end{abstract}

DOI: $10.21883 /$ FTP.2019.03.47289.9015

\section{1. Введение}

Квантово-каскадные лазеры (ККЛ), излучающие на нескольких длинах волн [1,2] применяются в ряде областей, основные из которых: формирование излучения на разностной частоте $[3,4]$, формирование излучения с переключением длины волны излучения [5], формирование излучения в широком спектральном диапазоне [6-8], формирование источника инфракрасного излучения с значительной перестройкой длины волны излучения $[9,10]$, для изучения и реализации эффекта четырехволнового смешения [11], создания систем газоанализа [12].

Несмотря на значительный прогресс за рубежом в области формирования ККЛ, излучающих на нескольких длинах волн, в РФ данные исследования находятся на начальном этапе. Результаты по выращиванию гетероструктуры двухчастотного ККЛ представлены в работе [13]. Результаты по двухчастотной генерации гетероструктур ККЛ на основе каскадов одного типа отражены в работах [14-16].

В настоящей работе представлены результаты по формированию и исследованию спонтанного и стимулированного излучения квантово-каскадных лазеров, изготовленных из гетероструктуры, сформированной методом молекулярно-пучковой эпитаксии (МПЭ) и состоящей из двух групп каскадов, которые излучают на разных длинах волн.

\section{2. Эксперимент}

Гетероструктура ККЛ была выращена компанией „Коннектор Оптикс“ с использованием промышленной установки МПЭ Riber 49, оснащенной твердотельным источником мышьяка крекерного типа и молекулярными источниками галлия и индия марки ABI 1000 на подложке InP с ориентацией (001), с проводимостью $n$-типа, легированной серой до уровня $5 \cdot 10^{16} \mathrm{~cm}^{-3}$.

При формировании гетероструктуры использована предложенная ранее конструкция с расширенным волноводом [13], включающая 36 квантовых каскадов, излучающих на длине волны 9.6 мкм и расположенных в центральной части волновода, в максимуме интенсивности световой волны с длиной волны 9.6 мкм. Квантовые каскады, которые излучают на длине волны 7.6 мкм, разделены на две части по 15 штук и расположены по бокам от квантовых каскадов, излучающих на длине волны 9.6 мкм, также вблизи двух максимумов световой волны для длины волны 7.6 мкм. Дополнительно, в волновод были введены слабо легированные $\left(n=2 \cdot 10^{16} \mathrm{~cm}^{-3}\right)$ слои $\mathrm{In}_{0.53} \mathrm{Ga}_{0.47} \mathrm{As}$, которые располагались с двух сторон от областей с квантовыми каскадами. Один, с толщиной 2100 нм, со стороны подложки и второй, с толщиной 1950 нм, со стороны поверхности гетероструктуры. В качестве нижней обкладки волновода была использована подложка из InP. Верхняя обкладка волновода была изготовлена из твердого раствора $\mathrm{In}_{0.52} \mathrm{Al}_{0.48} \mathrm{As}$, легированного кремнием до уровня $\mathrm{n}=1 \cdot 10^{17} \mathrm{~cm}^{-3}$, и имела толщину 2.0 мкм. Контактные слои из $\operatorname{In}_{0.53} \mathrm{Ga}_{0.47} \mathrm{As}$, ле- 
гированного до уровня $1 \cdot 10^{17} \mathrm{~cm}^{-3}$ и $1 \cdot 10^{19} \mathrm{~cm}^{-3}$, имеющие толщины 100 и 20 нм соответственно, завершали гетероструктуру. Суммарная толщина эпитаксиальных слоев гетероструктуры составила 10.3 мкм.

Две группы каскадов, которые излучают на длине волны 7.6 мкм, содержащие по 15 каскадов каждая, с суммарной толщиной 790.5 нм каждая, были изготовлены на основе гетеропары $\operatorname{In}_{0.53} \mathrm{Ga}_{0.47} \mathrm{As} / \mathrm{In}_{0.52} \mathrm{Al}_{0.48} \mathrm{As}$ и построены по схеме с двухфононным опустошением нижнего уровня $[17,18] .36$ каскадов, которые излучали на длине волны 9.6 мкм, с суммарной толщиной 2487.6 нм, были построены по схеме с трехфононным опустошением нижнего уровня [19].

Структурное качество гетероструктуры исследовалось методом рентгенодифракционного анализа и просвечивающей электронной микроскопии (ПЭМ). Измерения спектров рентгеновской дифракции с высоким разрешением были проведены вблизи симметричного рефлекca (004) InP на дифрактометре PANalytical X'PertPro в параллельной геометрии пучка рентгеновского излучения. В качестве источника излучения (мощностью 6 кВт) использована трубка с вращающимся медным анодом $(\lambda=0.15406$ нм). Полуширина первичного пучка не превышала $12^{\prime \prime}$, что обеспечивалось использованием четырехкратного Ge (220) прорезного кристалламонохроматора. ПЭМ исследования проводились на просвечивающем электронном микроскопе JEM2100F с ускоряющим напряжением 200 кВ. Образцы в геометрии поперечного сечения были подготовлены по стандартной методике путем скола структур по плоскостям (110) и дальнейшей шлифовки. На конечном этапе образцы утонялись путем распыления ионами $\mathrm{Ar}^{+}$ при энергиях 3-4 кэВ. Для определения границ между слоями получены изображения в двулучевых условиях с действующим вектором дифракции $g=(002)$, чувствительным к изменению химического состава. Далее был построен профиль интенсивности изображения, ортогональный границам слоев. Ширина усреднения составляла 20 нм. Положение границ между слоями принималось на полувысоте переходного контраста от одного слоя к другому.

Для исследования спектров спонтанного излучения были сформированы лазеры в четырехсколотой геометрии аналогично подходу, представленному в работе [20]. Геометрические размеры лазеров составляли $300 \times 300$ мкм. Для исследования спектров лазерной генерации были изготовлены полосковые лазеры. Формирование полоска лазера начиналось с вытравливания глубокой мезы жидкостным травлением (на основе раствора $\left.\mathrm{HBr}: \mathrm{HNO}_{3}: \mathrm{H}_{2} \mathrm{O}\right)$. В качестве маски для травления полоска использовался слой оксида кремния $\left(\mathrm{SiO}_{2}\right)$, локально вытравленный во фторсодержащей плазме под маской фоторезиста AZ5214. После формирования профиля мезы и удаления маски на основе $\mathrm{SiO}_{2}$ производилась пассивация боковых стенок мезы путем напыления слоя нитрида кремния, $\mathrm{Si}_{3} \mathrm{~N}_{4}$ толщиной 300 нм методом плазмохимического осаждения.

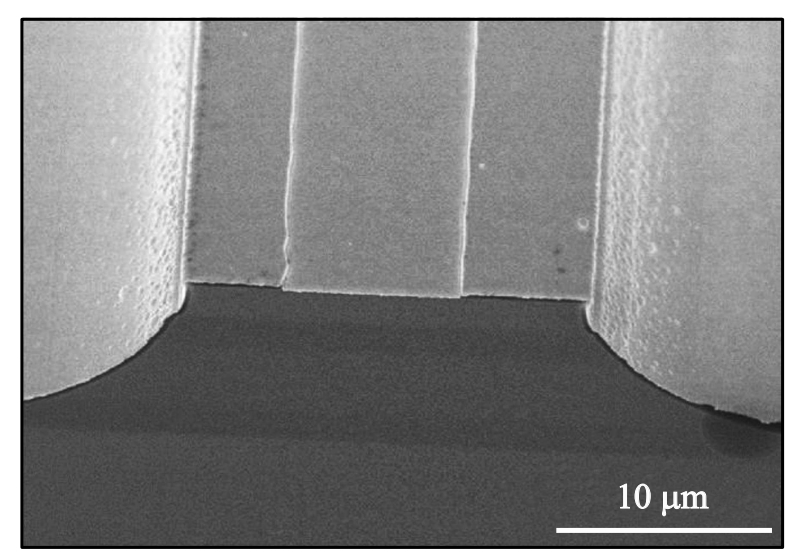

Рис. 1. Изображение скола изготовленного полоскового ККЛ, полученное методом сканирующей электронной микроскопии.

Вскрытие окон в слое $\mathrm{Si}_{3} \mathrm{~N}_{4}$ под металлизацию проводилось путем реактивного ионного травления в смеси газов $\mathrm{CHF}_{3} / \mathrm{O}_{2}$ под маской из фоторезиста. Верхняя металлизация формировалась за счет высоковакуумного напыления (через маску фоторезиста AZ2070) последовательных слоев титана и золота (Ti/Au) толщинами 20 и 200 нм соответственно. Перед напылением нижнего металлического контакта пластина утонялась до толщины 150 мкм. В качестве нижней металлизации использовалась последовательность слоев титан/золото с толщинами 4 и 200 нм соответственно. Зеркала лазеров формировались скалыванием. Ширина полоска составила 32 мкм, ширина окна, открытого в слое диэлектрика, $\sim 8$ мкм, длина резонатора была равна 1.5 мм. Изображение скола изготовленного ККЛ, полученное методом сканирующей электронной микроскопии, представлено на рис. 1. Отражающее и антиотражающее покрытия на сколотые грани лазера не наносились. Монтаж производился гетероструктурой вверх на посеребренный медный теплоотвод.

Четырехсколотые образцы на теплоотводе монтировались на медный держатель внутри заливного азотного криостата с выходным оптическим окном из селенида цинка. Криостат размещался так, чтобы торец лазера находился в фокусе входного параболического зеркала фурье-спектрометра Bruker Vertex 80v. Токовая накачка структуры осуществлялась импульсами длительностью 300 нс на частоте 5 кГц с помощью импульсной схемы на основе мощного полевого транзистора.

Детектирование оптического сигнала производилось с помощью фотоприемника $\mathrm{HgCdTe}$, охлаждаемого жидким азотом. Сигнал фотоприемника измерялся импульсным синхронным детектором SR250 с синхронизацией по импульсам тока и многократным усреднением. Измерение спектров производилось в режиме пошагового сканирования, интегральный оптический сигнал измерялся отдельно в хвосте интерферограммы. Вольтамперные характеристики (BAX) измерялись при про- 


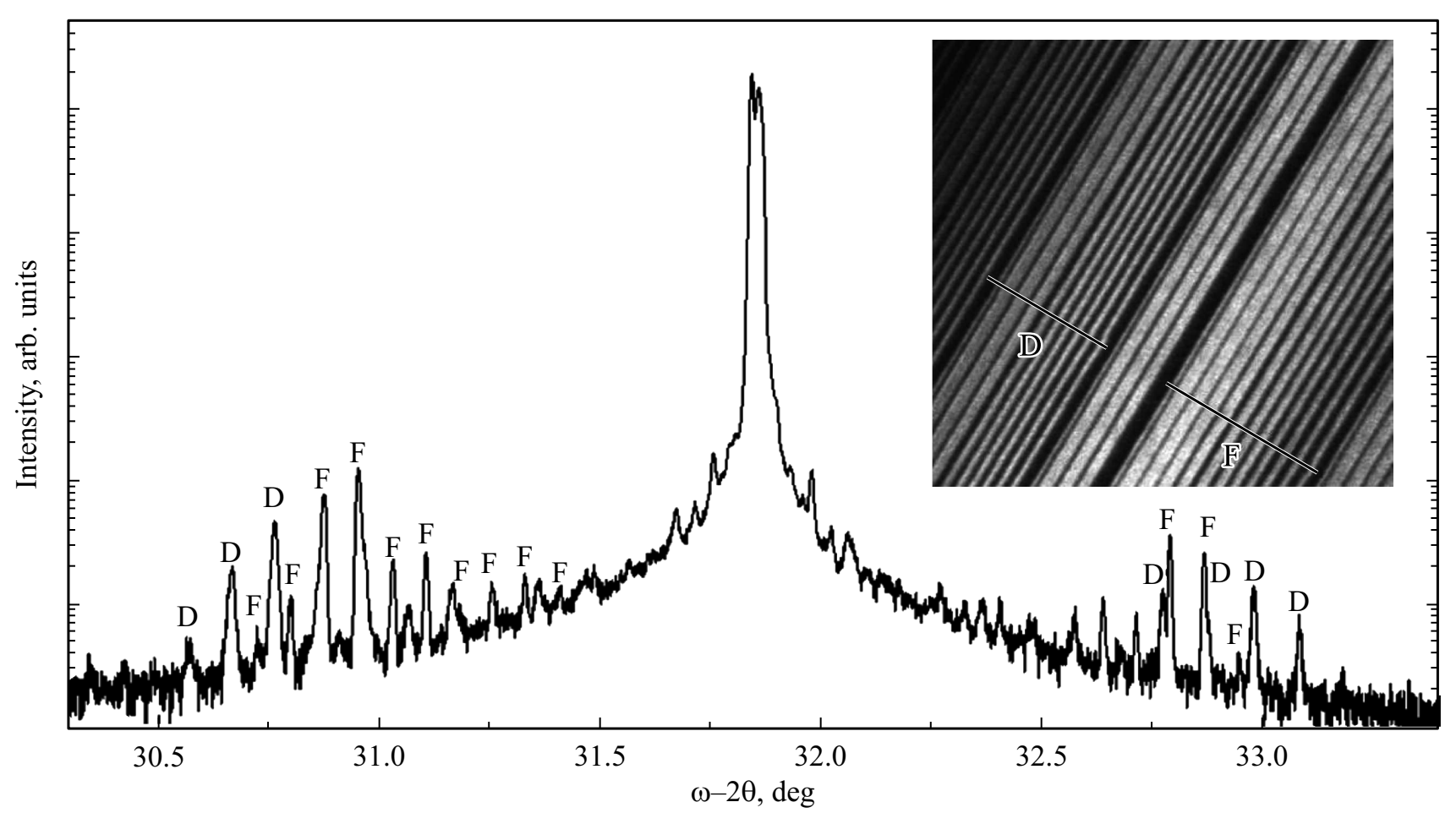

Рис. 2. Рентгенодифракционная кривая гетероструктуры двухчастотного ККЛ (в полулогарифмическом масштабе). На вставке изображение скола гетероструктуры, полученное методом ПЭМ.

пускании импульсов тока с длительностью 100 нс на частоте 84 кГц. Ватт-амперные характеристики измерялись с помощью калиброванного измерителя мощности Ophir 3A-FS. Все измерения проводились при температуре $78 \mathrm{~K}$.

\section{3. Результаты и их обсуждение}

Рентгенодифракционная кривая сформированной гетероструктуры представлена на рис. 2. Спектр демонстрирует выраженный максимум, соответствующий дифракционному пику от подложки, а также от толстых слоев верхней обкладки [13]. Продемонстрировано наличие двух различных последовательностей пиков-сателлитов, соответствующих каскадам двух типов, рассчитанных на длины волн генерации 9.6 и 7.6 мкм. Положение пиковсателлитов на дифракционной кривой двухчастотного ККЛ соответствует положению пиков для случая одночастотных ККЛ. В ряде случаев наблюдается наложение пиков-сателлитов, соответствующих каскадам активной области на 9.6 мкм, с пиками, соответствующими каскадам активной области на 7.6 мкм, что приводит к наличию пиков-сателлитов асимметричной формы. Обозначение „D“ пиков-сателлитов на рис. 2 соответствует периоду $52 \pm 1$ нм, что соотносится с данными толщины каскада активной области с длиной волны генерации 7.6 мкм, которая, согласно расчету, должна составлять 52.7 нм. Типичная ширина на полувысоте $(F W H M)$ данных пиков-сателлитов составляет $55^{\prime \prime}$. В свою очередь обозначение „Е“ $\mathrm{F}^{\text {с }}$ пиков-сателлитов соответствует толщине каскадов в $67 \pm 1$ нм. Толщина каскада активной области с длиной волны генерации 9.6 мкм, согласно расчету, должна составлять 69.1 нм. Типичные значения $F W H M$ данных пиков-сателлитов составляют $38^{\prime \prime}$. На вставке рис. 2 показано изображение каскадов гетероструктуры, полученное методом ПЭМ. Символами D и F отмечены каскады, соответствующие тем же обозначениям, которые приведены на спектре рентгеновской дифракции. Слои, входящие в каскады, отмечены пересекающими черными линиями. Исследование гетероструктуры методом ПЭМ показало соответствие выращенных слоев ростовому дизайну. Полные толщины двух типов каскадов, определенные на основе анализа ПЭМ-изображений, составили 68.7 и 51.0 нм соответственно. Приборная погрешность, согласно методике измерений, составляет $\pm 5 \%$. Протяженных дефектов не обнаружено. Все слои имеют планарные гетерограницы. Таким образом, результаты рентгеноструктурного анализа и просвечивающей микроскопии подтверждают высокое структурное совершенство изготовленных гетероструктур.

Спектры спонтанного излучения четырехсколотых образцов ККЛ, измеренные при различных уровнях электрической накачки представлены на рис. 3. В спектрах наблюдается несколько линий электролюминесценции (ЭЛ). При увеличении накачки вплоть до 2.5 А интенсивность ЭЛ на длине волны излучения 7.6 мкм возрастает, однако при дальнейшем увеличении тока до $3.1 \mathrm{~A}$ ин- 
тенсивность люминесценции на длине волны излучения 7.6 мкм начинает падать, при этом линия на длине волны излучения 9.6 мкм начинает разгораться. Таким образом, в спектре усиления одновременно наблюдается излучение с длинами волн 9.6 и 7.6 мкм, которое обеспечивается каскадами различной конструкции.

Спектр лазерной генерации полоскового лазера представлен на рис. 4. Продемонстрирована многомодовая лазерная генерация вблизи длины волны 7.6 мкм. Межмодовое расстояние между продольными модами $\Delta \lambda$, которое определяется длиной лазера, составило $\sim 1.1 \mathrm{~cm}^{-1}$. Отметим, что несмотря на то что в спектре усиления наблюдалась вторая линия вблизи длины волны 9.6 мкм, генерации на расчетной длине волны излучения 9.6 мкм не удалось достичь.

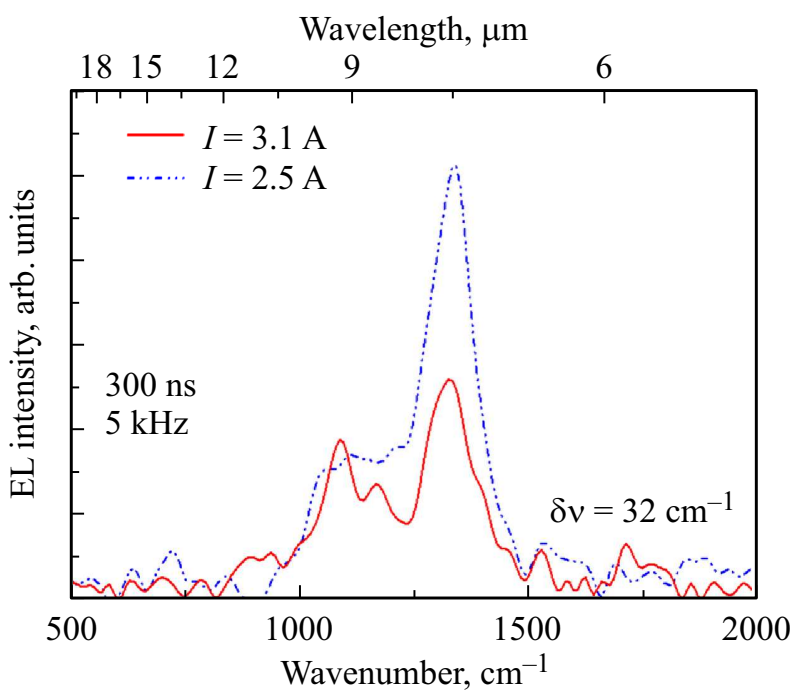

Pис. 3. Спектры спонтанного излучения четырехсколотого ККЛ. Сплошная линия соответствует току накачки $3.1 \mathrm{~A}$, штрихпунктирная линия - току накачки $2.5 \mathrm{~A}$.

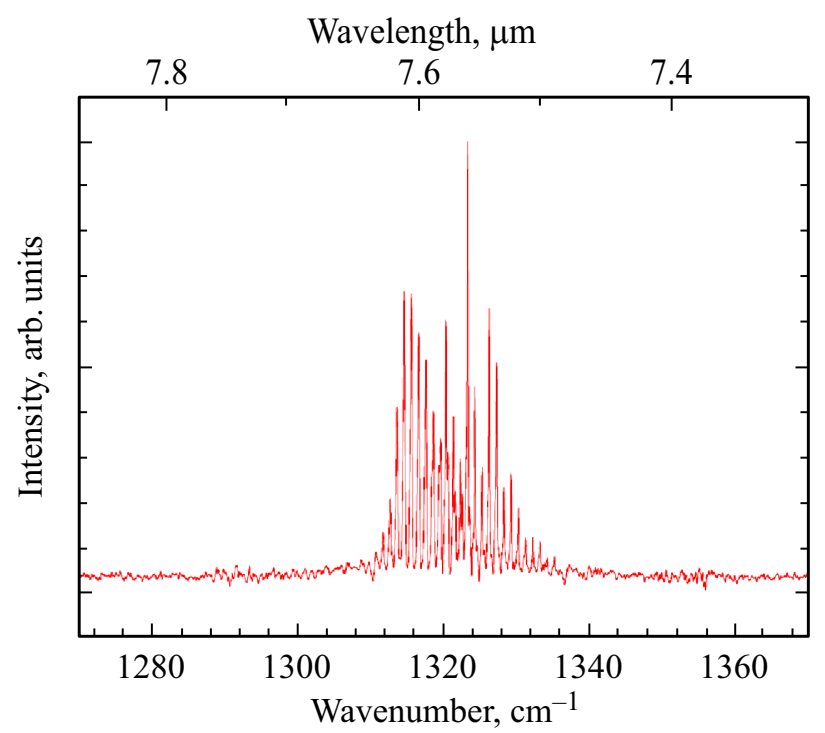

Рис. 4. Спектр лазерной генерации полоскового ККЛ.

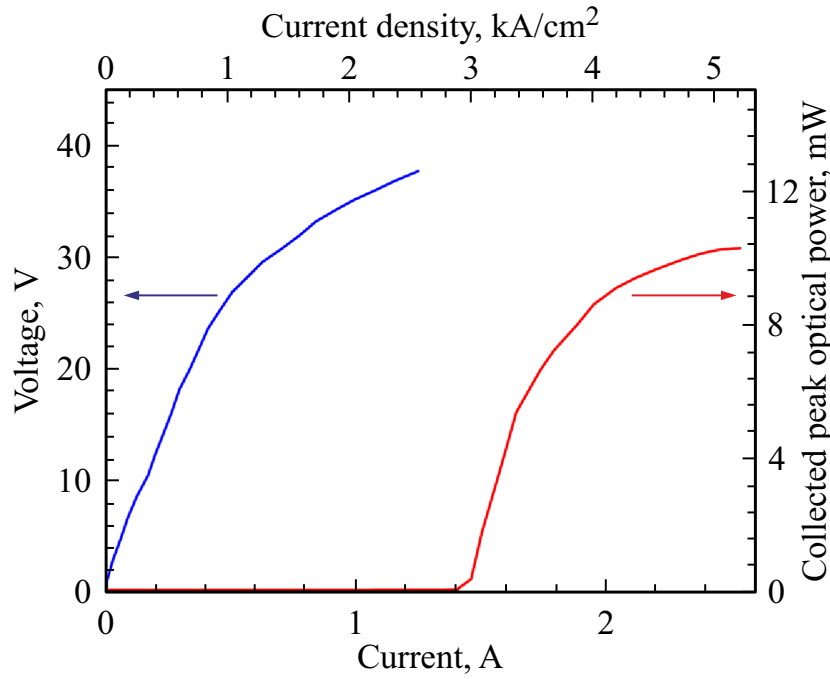

Рис. 5. Вольт-, ватт-амперные характеристики полоскового ККЛ.

Измеренные вольт-, ватт-амперные характеристики полоскового ККЛ представлены на рис. 5. Пороговое напряжение составило $\sim 38 \mathrm{~B}$, пороговый ток $\sim 1.4 \mathrm{~A}$, что соответствует пороговой плотности тока $\approx 2.8 \kappa \mathrm{A} / \mathrm{cm}^{2}$. Измеренная калиброванным датчиком выходная оптическая мощность (без корректировки на коэффициенты пропускания окна криостата на основе $\mathrm{Ge}$, линз на основе $\mathrm{Ge}$ и $\mathrm{ZnSe})$, с одного торца лазера, составила $\sim 10 \mathrm{MB}$.

\section{4. Заключение}

В ходе работы продемонстрирована лазерная генерация полосковых лазеров, изготовленных из многопериодной гетероструктуры двухчастотного квантовокаскадного лазера на основе гетеропары $\operatorname{In}_{0.53} \mathrm{Ga}_{0.47} \mathrm{As} /$ $\mathrm{Al}_{0.48} \mathrm{In}_{0.52} \mathrm{As}$. В качестве технологического метода выращивания гетероструктуры была применена молекулярно-пучковая эпитаксия. Спектр спонтанного излучения демонстрирует несколько пиков, соответствующих вкладу двух типов каскадов с длиной волны излучения 7.6 и 9.6 мкм. Однако лазерная генерация продемонстрирована только на длине волны излучения 7.6 мкм. Плотность порогового тока в полосковых лазерах составила $j_{\text {th }} \sim 2.8 \kappa \mathrm{A} / \mathrm{cm}^{2}$. Выходная оптическая мощность с одного торца лазера составила $\sim 10 \mathrm{MB}$. Дальнейшая оптимизации конструкции ККЛ с целью демонстрации генерации на двух длинах волн излучения может быть связана с использованием конструкции гетерогенных активных областей [10], активной области на основе одного типа квантовых каскадов с одновременными переходами на двух частотах [21], а также детальном расчете напряженностей полей в гетероструктуре на основе двух конструкций активных областей, с различным опустошением нижнего уровня [22]. 
Работа выполнена при финансовой поддержке РФФИ в рамках научного проекта № 16-29-09580. Электронномикроскопические исследования выполнены с использованием оборудования ЦКП „Материаловедение и диагностика в передовых технологиях“ (ФТИ им. А.Ф. Иоффе, Санкт-Петербург), поддержанного Министерством образования и науки России (Уникальный идентификатор проекта RFMEFI62117X0018).

\section{Список литературы}

[1] A. Straub, C. Gmachl, D.L. Sivco, A.M. Sergent, F. Capasso, A.Y. Cho. Electron. Lett., 38 (12), 565 (2002).

[2] K.J. Franz, D. Wasserman, A.J. Hoffman, D.C. Jangraw, K.-T. Shiu, S.R. Forrest, C. Gmachl. Appl. Phys. Lett., 90 (9), 091104 (2007).

[3] L. Consolino, S. Jung, A. Campa, M. De Regis, S. Pal, J.H. Kim, K. Fujita, A. Ito, M. Hitaka, S. Bartalini, P. De Natale, M.A. Belkin, M.S. Vitiello. Sci. Adv., 3(9), e1603317 (2017).

[4] M. Razeghi, Q.Y. Lu. Proc. SPIE, 9934, 993406 (2016). https://doi.org/10.1117/12.2240398

[5] A. Jiang, S. Jung, Y. Jiang, K. Vijayraghavan, J.H. Kim, M.A. Belkin. AIP Adv., 8 (8), 085021 (2018).

[6] C. Gmachl, D.L. Sivco, R. Colombelli, F. Capasso, A.Y. Cho. Nature, 415 (6874), 883 (2002).

[7] F. Toor, S.S. Howard, D.L. Sivco, C.F. Gmachl. IEEE J. Quant. Electron., 45 (8), 914 (2009).

[8] Y. Bidaux, R. Terazzi, A. Bismuto, T. Gresch, S. Blaser, A. Muller, J. Faist. J. Appl. Phys., 118 (9), 093101 (2015).

[9] F. Kapsalidis, M. Shahmohammadi, M.J. Süess, J.M. Wolf, E. Gini, M. Beck, M. Hundt, B. Tuzson, L. Emmenegger, J. Faist. Appl. Phys. B, 124 (6), (2018).

[10] W. Zhou, N. Bandyopadhyay, D. Wu, R. McClintock, M. Razeghi. Sci. Rep., 6 (1), (2016).

[11] A. Hugi, G. Villares, S. Blaser, H.C. Liu, J. Faist. Nature, 492 (7428), 229 (2012).

[12] M. Süess, P. Hundt, B. Tuzson, S. Riedi, J. Wolf, R. Peretti, M. Beck, H. Looser, L. Emmenegger, J. Faist. Photonics, 3 (2), 24 (2016).

[13] A.V. Babichev, A.S. Kurochkin, E.C. Kolodeznyi, A.V. Filimonov, A.A. Usikova, V.N. Nevedomsky, A.G. Gladyshev, L.Y. Karachinsky, I.I. Novikov, A.Y. Egorov. Semiconductors, 52 (6), 745 (2018).

[14] V.V. Dudelev, S.N. Losev, V.Yu. Mylnikov, A.V. Babichev, E.A. Kognovitskaya, S.O. Slipchenko, A.V. Lutetskii, N.A. Pikhtin, A.G. Gladyshev, L.Ya. Karachinskii, I.I. Novikov, A.Yu. Egorov, V.I. Kuchinskii, G.S. Sokolovskii. Opt. Spectrosc., 125 (3), 402 (2018).

[15] V.V. Dudelev, S.N. Losev, V.Yu. Myl'nikov, A.V. Babichev, E.A. Kognovitskaya, S.O. Slipchenko, A.V. Lyutetskii, N.A. Pikhtin, A.G. Gladyshev, L.Ya. Karachinsky, I.I. Novikov, A.Yu. Egorov, V.I. Kuchinskii, G.S. Sokolovskii. Tech. Phys., 63 (11), 1656 (2018).

[16] V.V. Dudelev, S.N. Losev, V.Yu. Mylnikov, A.V. Babichev, E.A. Kognovitskay, S.O. Slipchenko, A.V. Lyutetsky, N.A. Pikhtin, A.G. Gladyshev, L.Ya. Karachinsky, I.I. Novikov, A.Yu. Egorov, V.I. Kuchinsky, G.S. Sokolovskii. Phys. Solid State, 60 (11), 2291 (2018).
[17] A.V. Babichev, A.G. Gladyshev, A.V. Filimonov, V.N. Nevedomskii, A.S. Kurochkin, E.S. Kolodeznyi, G.S. Sokolovskii, V.E. Bugrov, L.Y. Karachinsky, I.I. Novikov, A. Bousseksou, A.Y. Egorov. Techn. Phys. Lett., 43 (7), 666 (2017).

[18] A.V. Babichev, A.G. Gladyshev, A.S. Kurochkin, E.S. Kolodeznyi, G.S. Sokolovskii, V.E. Bougrov, L.Y. Karachinsky, I.I. Novikov, A.G. Bousseksou, A.Y. Egorov. Semiconductors, 52 (8), 1082 (2018).

[19] A.V. Babichev, S.A. Gusev, A.N. Sofronov, D.A. Firsov, L.E. Vorob'ev, A.A. Usikova, Yu.M. Zadiranov, N.D. Il'inskaya, V.N. Nevedomskii, V.V. Dudelev, G.S. Sokolovskii, A.G. Gladyshev, L.Ya. Karachinskii, I.A. Novikov, A.Yu. Egorov. Techn. Phys., 63 (10), 1512 (2018).

[20] A.Y. Egorov, A.V. Babichev, L.Y. Karachinsky, I.I. Novikov, E.V. Nikitina, M. Tchernycheva, A.N. Sofronov, D.A. Firsov, L.E. Vorobjev, N.A. Pikhtin, I.S. Tarasov. Semiconductors, 49 (11), 1527 (2015).

[21] K. Fujita, M. Hitaka, A. Ito, T. Edamura, M. Yamanishi, S. Jung, M.A. Belkin. Appl. Phys. Lett., 106 (25), 251104 (2015).

[22] M.A. Belkin, F. Capasso, A. Belyanin, D.L. Sivco, A.Y. Cho, D.C. Oakley, C.J. Vineis, G.W. Turner. Nature Photonics, 1 (5), 288 (2007)

Редактор А.Н. Смирнов

\section{Spontaneous emission and lasing of two-wavelength quantum-cascade laser}

\author{
A.V. Babichev ${ }^{\mathbf{1}, 2}$, A.G. Gladyshev ${ }^{\mathbf{1}, 2}$, A.S Kurochkin ${ }^{\mathbf{1}}$, \\ E.S. Kolodeznyi ${ }^{1}$, V.N. Nevedomskii ${ }^{3}$, \\ L.Ya. Karachinsky ${ }^{1,2,3}$, I.I. Novikov ${ }^{1,2,3}$, \\ A.N. Sofronov ${ }^{4}, A . Y u$. Egorov ${ }^{1,2}$ \\ ${ }^{1}$ ITMO University, \\ 197101 St. Petersburg, Russia \\ ${ }^{2}$ Connector Optics LLC, \\ 194292 St. Petersburg, Russia \\ ${ }^{3}$ loffe Institute, \\ 194021 St. Petersburg, Russia \\ ${ }^{4}$ Peter the Great St. Petersburg Polytechnic University, \\ 195251 St. Petersburg, Russia
}

\begin{abstract}
Experimental results on the fabrication and study of a two-wavelength quantum-cascade laser based on a system of $\mathrm{In}_{0.53} \mathrm{Ga}_{0.47} \mathrm{As} / \mathrm{Al}_{0.48} \mathrm{In}_{0.52} \mathrm{As}$ solid alloys on an InP substrate by using molecular beam epitaxy are presented. The heterostructures contained quantum cascades emitting at a wavelength of $9.6 \mu \mathrm{m}$, and cascades that emit at a wavelength of $7.6 \mu \mathrm{m}$. The high structural quality of the fabricated heterostructures is shown. The spectra of spontaneous and stimulated emission were studied. Multimode lasing of edge-emitting lasers at a wavelength of $7.6 \mu \mathrm{m}$ was demonstrated.
\end{abstract}

